\title{
Control and Information Architectures for Formations
}

\author{
Brian D.O. Anderson, Changbin Yu, Barış Fidan, Julien M. Hendrickx
}

\begin{abstract}
This paper reviews a number of concepts and results relevant to the design of architectures to maintain the shape of a formation of autonomous agents. The paper begins with motivating examples from nature and the manmade world, and emphasises the task of providing satisfactory sensing, communication and control architectures within a formation of autonomous agents. Then some technical tools for characterising and designing architectures are described, largely resting on graph theoretic considerations.
\end{abstract}

\section{INTRODUCTION}

For millennia if not millions of years, nature has presented examples of collective behaviour by groups of insects, birds, fish, etc. Such behaviour has arisen to permit sophisticated behaviour of the group that would never be achievable by individual members of the group [19], [22]. The behaviour may serve the needs of foraging for food, of defence against predators, of aggression against prey, of mating, etc. Fish and birds particularly, as part of their group behaviour, often display formation type behaviour; in this sort of behaviour the relative positions of the fish or birds are preserved, at least more or less, and the whole formation moves as a cohesive whole. Of course, from time to time, a formation may split, rearrange itself in a minor way perhaps to remove a burden on one or members of the formation, rearrange itself in a major way, perhaps for obstacle avoidance or predator avoidance, merge with another formation, etc.

Whether nature has been a conscious or unconscious motivator for human kind, formations of robots, underwater vehicles and autonomous airborne vehicles are now slowly being deployed to tackle problems in both civilian and military spheres-bush fire control, surveillance, underwater exploration and the like. A formation of vehicles may constitute a much more effective sensor than a single vehicle, for various reasons. First, having many vehicles allows effective synthesis of a large dimension antenna, for receiving electromagnetic or acoustic signals, and large antennas allow

B.D.O. Anderson, C. Yu and B. Fidan are with National ICT Australia Ltd. and Research School of Information Science and Engineering, The Australian National University, Canberra ACT 2061, Australia, and they are supported by National ICT Australia, which is funded by the Australian Government's Department of Communications, Information Technology and the Arts and the Australian Research Council through the Backing Australia's Ability Initiative. C. Yu was an Australia-Asia Scholar supported by the Australian Government's Department of Education, Science and Training through Endeavours Programs. $\{$ Brian. Anderson, Brad.Yu, Baris.Fidan\}enicta.com.au

Julien M. Hendrickx is with Department of Mathematical Engineering, Universit Catholique de Louvain and is a Belgian Fund for Scientific Research (FNRS) fellow. His work is supported by the Concerted Research Action (ARC) "Large Graphs and Networks" of the French Community of Belgium and by the Belgian Programme on Interuniversity Attraction Poles initiated by the Belgian Federal Science Policy Office. The scientific responsibility rests with its authors.Hendrickx@inma.ucl.ac.be better source localization, or simply have higher sensitivity; accurate knowledge and control of the relative positions of the agents of the formation is of course essential for this application. A second reason is that some tasks inherently require multiple sensors of known relative positions; for example, in three dimensions, if one can measure distances to an object of interest, and if one wants to determine the position of that object, one needs four separate distance measurements from sensors with known positions to determine that object's position (otherwise termed localizing the object). A third reason is that multiple sensors may have individually differing functionalities, which in aggregate gives a new functionality for the formation of sensors. Small mobile sensors are much cheaper to deploy, but then weight and other considerations will set upper bounds on the on-board functionality of any one sensor.

Sometimes these factors apply at the same time. For example, in one application with which the authors are familiar, the agents of the formation are localizing by determining angle information, but issues of sensor noise, limited cone of visibility and the like, mean that more agents need to be used in the formation that might be at first thought. Formations in naturally or artificially hazardous environments also may require larger numbers of agents, to cope with outages.

From a control point of view, it is clear that there are tasks at both the level of the whole formation, determining for example waypoints for a path which the centre of gravity of the formation should follow, as well as control tasks for the individual agents of the formation, such as maintaining their relative positions, or shifting from one formation shape to another formation shape. Certainly in formations occurring in nature, and commonly in man made formations, there is no single all-powerful master agent exercising control over every other agent. Control tasks in some way have to be handled on a decentralized basis.

In fact many systems problems arise, and standing at the apex of these problems is the task of defining practical architectures for control, communications and sensing. Such architectures of course cannot be defined independently of one another. An overarching requirement is that architectures be scalable. The scalability requirement imposes a need for significant decentralization of information and control structures, and, just as in a formation of birds, no one bird can be expected to watch all other birds and compute its own trajectory using even partial knowledge of the trajectories of all other individual birds, so the amount of sensing, communication and control computation by any one agent has to be limited.

We have sketched a vast problem domain above. This 
paper will not traverse the whole problem domain, but only one corner, albeit an important corner. Most of this paper will describe what sort of sensing and control architectures are needed to maintain the shape of a formation, while the formation moves as a cohesive whole. As already intimated, in many situations, it is desirable that the formation maintain its shape while executing an overall change of location, or change from one shape to another, perhaps to avoid an obstacle; again, on occasions a formation may need to split, or merge with a different formation. The architectures need to be able to sustain these tasks.

Within this limited domain, there is a further limitation we will impose. Obviously, to maintain a formation shape, agents have to sense some aspect of the formation geometry, i.e. an agent will need to measure some geometricallyrelevant variable involving some, at least, of some other agents in the formation, in order to apply a control to correct any error in formation shape. There are many things that can be sensed, involving distances and angles, for example agent $j$ might sense

- The distance from agent $j$ to agent $k$

- The difference in the distance from agent $j$ to agent $k$ and the distance from agent $j$ to agent $m$ ("time difference of arrival")

- The bearing relative to north of agent $k$

- The declination/inclination relative to the horizon of agent $k$

- The angle subtended at agent $j$ by the lines joining agent $j$ to agent $k$ and agent $k$ to agent $m$

etc. We note that biological organisms may sense more complicated things again than the above, and matters are far from clear anyway. One of our colleagues conjectures that birds effectively project angles within a cone onto a hemisphere, and at the same time sense perhaps one distance (so that the formation is not scale free, which would be the case were angles alone to be sensed). In this paper, we shall confine our attention to the use of distance measurements.

Many of our tools will be graph theoretic. We will not be presenting control laws for individual agents in a formation, but focussing on the higher-level question of defining the architecture behind these control laws. There has been considerable work on control laws, see e.g. [2], [4], [11], [21], [24], [25], [29]. Though not discussed here, it turns out that the graph theoretic tools we describe can often be applied to formations with other sensed variables than just distance, including those with mixtures of angles and distances, see e.g. [9].

\section{OUTLINE OF THE PAPER}

In the next two sections, we describe how aspects of formation architecture can be described using graphs. There is a clear dividing line between the two sections that is associated with the sort of graphs we are using. Consider two agents in a formation, $j$ and $k$. Suppose that the distance between these agents is to be actively maintained, and that one or both of the agents in question can sense that distance. Then the task of maintaining the distance might be one that is given jointly to $j$ and $k$ (in which case both would have to sense the distance). Alternatively, it might be given to $j$ alone, or $k$ alone (in which case only one would need to sense the distance); if it were given to $j$ alone, then $k$ would be unconscious of $j$. In the former instance, with joint responsibility, it turns out that undirected graphs are an appropriate tool (and the graph will have an undirected edge between vertices corresponding to agents $j$ and $k$ ). In the latter case (responsibility given to $j$ alone or $k$ alone), the graphs are directed, and the graph will have a directed edge from $j$ to $k$ (when $j$ is responsible for maintaining distance) or from $k$ to $j$ (when $k$ is responsible for maintaining distance).

These two sections describe conditions, in two and three dimensions, that must be fulfilled by architectures that allow preservation of formation shape during formation movement.

In Section $\mathrm{V}$, we address operations with formations, including merging, splitting and closing ranks. Closing ranks is the task of repairing a formation when one or more agents are lost; it is a task that obviously nature has solved in a number of cases. Generally speaking, a minor rearrangement of the architecture is needed.

Section VI develops these tasks in some more detail. There are two very broad conceptual approaches that can be adopted. Consider the merging problem. One can contemplate two formations, both capable of maintaining cohesive motion, and ask the question, if they are brought into proximity with one another and thought of as a single formation with agent set the union of the agent sets of the individual formations, what additional inter-agent distances might have to be sensed and controlled (beyond those already being sensed and controlled in the individual formations) in order that the new single formation be capable of cohesive motion? This is a very natural way to look at the problem. The second conceptual approach says: think of each of the two formations as a sort of super agent, or meta-agent, with an internal structure that is not fundamentally important. Then we need to identify the rules for assigning edges between two meta-agents in order that the combination be capable of maintaining cohesive motion. This view is a much more recent one.

In Section VII we offer some concluding remarks.

\section{FORMATIONS AND UNDIRECTED RIGID GRAPHS}

In this section, we indicate the applicability of graph theory to formations.

Rigid graph theory [18], [20], [31], [32] is a tool which has been used to analyse the property of formation rigidity, see [9], [25], [28], [37]. Agents are modelled as points. Agent pairs for which the inter-agent distance is actively constrained to be constant can be thought of as being joined by bars with lengths enforcing the inter-agent distance constraints. The system can be therefore modelled by a graph where vertices represent point-like agents and interagent distance constraints are abstracted as edges. (Naturally, one can contemplate other constraints than distance, e.g. those involving angle, or angle and distance; however the 
theory begins with distance constraints, and we will restrict discussion to this case). Rigid graph theory is concerned with stating properties of graphs which ensure that the formation being modelled by the graph will be rigid; formal definitions are available of course, but roughly speaking, a rigid formation is one in which the only smooth motions are those corresponding to translation or rotation of the whole formation.

Figure 1 shows several examples of two dimensional graphs, two of which are rigid and one of which is not rigid. In a nonrigid graph part of the graph can flex or move, while the rest of the graph stays still. The notion of rigidity conforms to one's normal intuition.

\section{A. Rigidity and Minimal Rigidity}

Two key tools of analysis appear. The first is a tool of linear algebra. Given knowledge of the positions of the agents at any one time, one can construct a matrix, the socalled rigidity matrix [31], [32], and the dimensions and rank of this matrix allow one to conclude that the formation is or is not rigid. The dimensions and rank are the same for almost all positions of the agents. This means that rigidity matrices formed from two formations differing from each other only in terms of the values for the constrained distances will have the same rank, except for very special sets of the agent positions. Appendix A contains more detail on the rigidity matrix. The concept is valid in both two and three dimensions.

The characterization of rigidity using the rigidity matrix rests on using linear algebra ideas, and yet, since rigidity is a property that will occur for almost all instantiations of a graph, i.e. for almost all vertex positions, except those lying in a nongeneric set, it is reasonable to conjecture that there should be a test for rigidity in which the particular values of the vertex positions need not appear. Indeed this is the case for two dimensions, but not yet for three dimensions.

It proves possible in two dimensions to also characterize rigidity in purely combinatorial terms, i.e. counting-type conditions related to the graph (discarding therefore the agent coordinates) can be used to conclude the rigidity or otherwise of a generic formation corresponding to the graph. This is the celebrated Laman's Theorem [23], for which no threedimensional equivalent exists. In three dimensions, differing necessity and sufficiency conditions are known for a graph to correspond to a formation which will be rigid for generic values of the constrained inter-agent distances [31]. For more detail see Appendix B.

One major result concerns the construction of rigid formations. As for the characterisation of rigidity, the theory for two-dimensional formations is better developed than that for three-dimensional formations. Before describing the result, we flag the concept of minimal rigidity. A formation is minimally rigid if it is rigid and if no single inter-agent distance constraint can be removed without losing rigidity. A graph is minimally rigid if almost all formations to which the graph corresponds are minimally rigid. Minimal rigidity is easily described in two and three dimensions with the rigidity

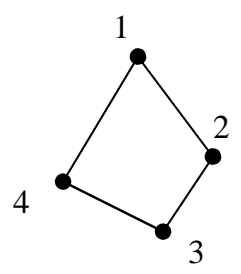

(a)

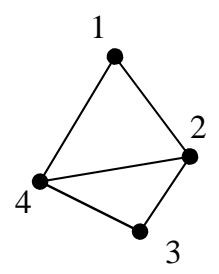

(b)

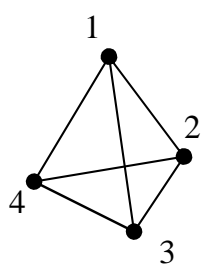

(c)
Fig. 1. Illustration of (a) non-rigid formation, (b) (minimally) rigid formation, and (c) non-minimally but rigid formation

matrix, characterisable in two dimensions with Laman's Theorem, and the subject of some necessary conditions in three dimensions on the graph determined by a formation. Necessary conditions in two and three dimensions are that $|E|=2|V|-3$ and $|E|=3|V|-6$ respectively, where $|E|$ and $|V|$ are the numbers of edges and vertices of the graph. See Figure 1 for an illustration.

The two-dimensional construction result referred to above is this. Suppose a minimally rigid graph corresponding to a two-dimensional formation exists. Then there are two operations on rigid formation construction known as the vertex addition operation and the edge-splitting operation which can be used to build another minimally rigid graph with one more vertex. Obviously, bigger and bigger minimally rigid graphs can be built this way, with the process being known as Henneberg sequence construction [7], [31]. What is important are the following two additional properties:

- All two-dimensional minimally rigid graphs with any number of vertices are constructible from a primitive comprising a two-vertex single-edge graph by an appropriate sequence of these operations, see Appendix C.

- Any two-dimensional minimally rigid graph can be "deconstructed" by the inverse operations, to yield a sequence of minimally rigid graphs each with one less vertex than its predecessor in the sequence, and terminating with a two-vertex, single-edge graph.

Figure 2 illustrates a Henneberg construction.

In three dimensions, the results are not so complete and it is still a matter of conjecture that a certain set of operations is necessary and sufficient to build and "deconstruct" all minimally rigid graphs. Some conjectures are provided in [31].

\section{B. Extension to the concept of Global Rigidity}

Before introducing the works reflecting the title of the paper, we need to digress to introduce another important graph theoretic concept, global rigidity. Consider a formation in which agents are labelled, and certain inter-agent distances are prescribed but the Euclidean positions of the agents are not known. One can then ask: what Euclidean positions of the agents would correspond to the data? Obviously translations and rotations must be allowed. Almost as obviously, reflections must be allowed. Thus if the agents were so located as to correspond to the data, and if then the signs of all 


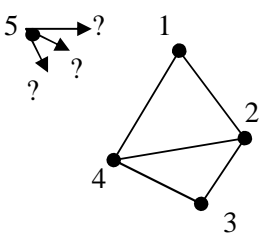

(a)
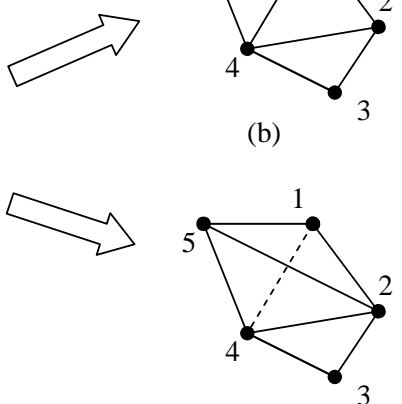

(c)
Fig. 2. Illustration of (a) growing a four agent formation to include agent 5 , by (b) vertex addition operation, OR, (c) edge splitting operation, where edge $\{1,4\}$ is the removed edge.

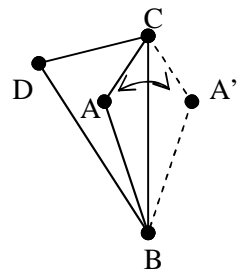

(a)

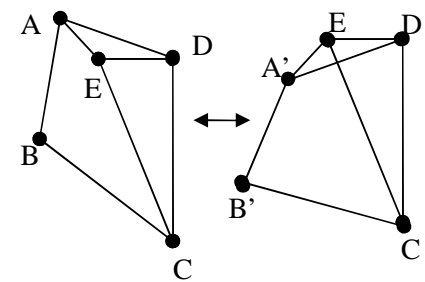

(b)
Fig. 3. Illustration of (a) Flip ambiguity: Vertex A can be flipped over the edge $(B, C)$ to a symmetric position $A^{\prime}$ and the distances constraints remain the same. (b) Discontinuous flex ambiguity: Temporarily removing the edge $(A, D)$, the edge triple $(A, E),(A, B),(B, C)$ can be flexed to obtain positions $A^{\prime}$ and $B^{\prime}$, such that the edge length $\left(A^{\prime}, D\right)$ equals the edge length $(A, D)$, therefore all the distance constraints are the same.

the coordinates of every agent were changed and new agents placed at the new positions, another formation corresponding to the distance data would result, and in general it would not be obtainable from the first by translation and rotation.

A two-dimensional or three-dimensional formation (and by extension its graph) is termed globally rigid if and only if any two formations corresponding to the distance data differ by at most translation, rotation and reflection. It is perhaps not immediately obvious, but global rigidity is a more demanding concept than rigidity, i.e. there exist rigid formations which are not globally rigid, and such formations can only be converted to globally rigid formations by the addition of more distance constraints. Figure 3 gives two examples of two dimensional formations which are rigid but not globally rigid, and yet correspond to the same set of distance constraints.

The notion of flip ambiguity is transparent. Not every minimally rigid graph contains a flip ambiguity. The notion of flex ambiguity needs more explanation. The two graphs of Figure 3(b) satisfy the same distance constraints, but are clearly quite different. Every minimally rigid graph with four or more vertices at generic positions can exhibit flex ambiguity (for the graph of Figure 3(a), the flex ambiguity happens to be the same as the flip ambiguity). This means that where there are only enough distance constraints to ensure minimal rigidity, the shape of an associated formation is not uniquely specified by those constraints, though if a formation assumes one of the allowed shapes, it cannot deform smoothly from that shape. Minimal rigidity allows retention of shape, but does not of itself specify what shape is retained. Global rigidity instead is required.

Appendix D contains some more remarks on global rigidity.

Henneberg sequence construction is also possible for twodimensional globally rigid graphs. Two different sets of operations have been advanced, see [7] and [20]. Recent unpublished work of some of the authors has shown that both sets of operations lead to the same graphs, in fact all globally rigid graphs can be grown this way.

The definition of global rigidity for two-dimensional formations extends obviously to three dimensions. However, though global rigidity is a generic concept in two dimensions, and thus is a concept that can be associated with a graph, it is not known whether it is a generic concept in three dimensions, although certainly some three-dimensional graphs can legitimately be termed globally rigid. What this means is that there may be two three dimensional formations with the same graph, but with different specified inter-agent distances, such that one of these formations has a shape not uniquely determined by the specified inter-agent distances (i.e. is rigid but not globally rigid) while the other formation has a shape that is uniquely determined by the specified interagent distances (i.e. it is globally rigid).

Global rigidity is of interest in various application areas, including sensor network localization [8], [12], [26]. In sensor networks, there is given a set of points (like the agents in a formation but corresponding to sensors), and a set of distances between pairs of points (obtained by exchange of information between points in the network); the distances are typically available for pairs of points which are within a Euclidean distance of one another that is less than some threshold. The associated graph is termed a unit disk graph. The sensor network localization problem is to pass from the distance set to a set of Euclidean coordinates for the sensors consistent with the distance set. In the absence of further information, the Euclidean coordinates are only specified up to translation, rotation or reflection. That further information is normally obtained from so-called anchor nodes or sensors, the position of which are known absolutely.

It turns out that a number of current applications of formations involve localization of objects whose position is unknown. Recently for example, the following applications problem (in $\Re^{2}$ ) was posed to us: Suppose there are the agents $A, B$ and $C$ at known positions, and three agents $D, E$ and $F$ at unknown positions. Suppose that the interagent distances are known for $A, B$ and $C$, and separately for $D, E$ and $F$. Suppose further that the distances $A D, B E$, and $C F$ are known. Can one localize $D, E$ and $F$, and if so, how? While we mention this problem simply to motivate the importance of studying localization, its solution being unimportant for the overall message of the paper, we can 
nevertheless record what the solution is: the associated graph can be shown to be minimally rigid, and so not globally rigid. Generically therefore, $D, E$ and $F$ cannot be localized, though a finite set of positions for each of $D, E$ and $F$ can be determined, the positions differing by flex ambiguities.

\section{Nonminimally rigid formations}

Globally rigid formations are nonminimally rigid. But are there other reasons for using nonminimally rigid formations, where more controls are imposed than are needed? And are there any special problems in doing so?

There are indeed good reasons to use nonminimally rigid graphs to underpin the shape of a formation. In a minimally rigid formation, there is no protection against loss of a sensor, a communication link or a control actuator, and in practice, it will often be necessary to obtain robustness through the use of some measure of redundancy. Measures of robustness are needed to reflect ability to sustain loss of an edge (whether from a sensing, communication or control failure), or the loss of an agent, or indeed the loss of a multiplicity of edges and/or agents. Loss of agents is in part covered by the discussion subsequently of closing ranks.

Are there any special problems in handling nonminimally rigid formations? There is one problem, but it can be overcome. Imagine a two-dimensional physical structure that is nonminimally rigid. If it were to be constructed from a plan, it is clear that if mistakes are made in determining the lengths of the members corresponding to the edges, then in some way the assembly of the structure will not fit together properly. Likewise, if distances between agents in a formation are measured with some noise, there will be some inconsistency. In $\Re^{2}$, an agent in a formation with three neighbours from which certain distances have to be maintained may not be able to consistently position itself so that all measured values are consistent with all the nominal values. This sort of problem, i.e. having to cope with inconsistencies introduced by inaccurate measurements, also arises in sensor network localization problems, and techniques are slowly becoming available to deal with this [3].

\section{Formations AND DIRECTED GRAPHS}

The discussion to this point about formation information and control architectures to secure rigidity has been sketchy. Let us observe now that the task of maintaining a prescribed distance between a nominated agent pair requires control action, and one can conceive that the execution of the task could be the "responsibility" of both agents, or one nominated agent of the pair. Modelling using undirected graphs is appropriate in the former case. However in the latter case, it is important to recognise the distinction by assigning a direction to all edges in the graph. A directed edge from vertex $u$ to vertex $v$ appears when agent $u$ has the task of maintaining its distance constant from agent $v$, and agent $v$ is unconstrained in its own motions with respect to the motion of $u$, i.e. it is "unconscious" of the task that agent $u$ has to execute.

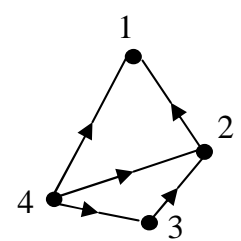

(a)

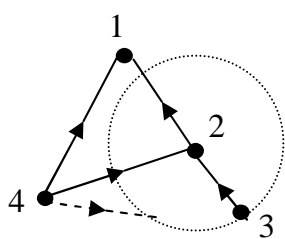

(b)
Fig. 4. Illustration of a 4-agent directed formation that is not constraint consistent

\section{A. Constraint Consistence and Persistence of Formations}

There is still interest in the basic question: what conditions ensure that the motions of a formation are restricted to translation or rotation? This question is examined in [13] for two dimensions, and in [14], [36], [37] for three dimensions. We will describe the two-dimensional result first. A notion termed persistence is introduced, which is an amalgam of two conditions, rigidity (as before) and a notion termed constraint consistence. The rigidity property says that if certain interagent distances are maintained, then all inter-agent distances are maintained when the formation moves smoothly. The new property, constraint consistence, is equivalent to the requirement that it is possible to maintain the nominated inter-agent distances. To illustrate this further, consider Fig. 4 above.

Suppose agents 1 and 2 are fixed, with agent 2 at its correct distance from 1. Suppose also that agent 3 is at its correct distance from agent 2 and agent 4 at its correct distance from 1, 2 and 3. Now observe that agent 3 has only one distance constraint, thus it can move, while maintaining its distance from 2 , on a circle centered at agent 2 . It is unconscious of the constraint which 4 is supposed to maintain on the distance between agents 3 and 4 . When agent 3 moves, agent 4 then has an impossible task. There are only two possible positions where agent 4 can be in order to maintain its correct distances from agents 1 and 2; for generic allowable positions of agent 3 , agent 4 will not be able to maintain the correct distance from agent 3 from either of these two positions. We describe such an arrangement as being not constraint consistent. Evidently, too much is being asked of one agent.

Constraint consistent formations are those where no agent is given potentially impossible constraints, in the manner of agent 4 in Fig. 4. The notion of constraint consistence can be applied and described with directed graphs. Formal definitions of constraint consistence and persistence can be found in [13], [37]. Let us simply note the following key facts:

- Any two dimensional graph which has no more than two outgoing edges from any vertex is constraint consistent (though there are constraint consistent graphs where some vertices have out-degree greater than two).

- A graph can be checked for persistence (i.e. rigidity plus constraint consistence) by testing a certain collection of subgraphs (in which the edge directions are neglected) for rigidity. See Appendix E for further remarks. 
(a)

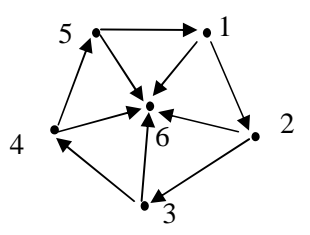

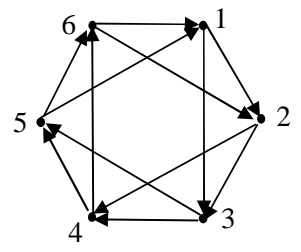

(b)
Fig. 5. Illustration of (a) a persistent wheel graph, and (b) a persistent $C^{2}$ graph

\section{B. Securing persistence: some examples}

In the light of the above remarks, an important question presents itself. Suppose that a two-dimensional undirected graph is rigid. Can one assign edge directions so that it is constraint consistent and thus persistent? At the time of writing, the question in its full generality remains open. However, affirmative answers exist for minimally rigid graphs [13], and graphs with certain structures, including wheel graphs, trilateration graphs, complete graphs and power graphs of circle graphs [10], [33].

The simplest algorithm for assigning directions in a minimally rigid graph is to consider the associated undirected graph and determine the Henneberg sequence whereby it can be grown. Then it is trivial to add directions at each step, simply using the rule that any vertex can have no more than two outgoing edges, see [13], [15] for details. We remark that such a directed graph is termed minimally persistent; minimally persistent graphs are precisely those which are minimally rigid and constraint consistent.

Figure 5 shows some direction assignments for wheel graphs and the graph known as $C^{2}$, these being two structures that at times have been advanced as being useful for autonomous agent formations. (The graph $C^{2}$ is so designated as it is the square in a graph theoretic sense of the cycle graph, usually designated by $C$ ). The structures have distinct robustness properties, i.e. tolerance of agent or link loss in the formation (corresponding to vertex or edge loss in the graph). A wheel graph with a total of $N$ vertices has $2 N-2$ edges, and can tolerate the loss of any single edge while still remaining persistent. Also it can tolerate the loss of any single vertex other than the 'central' vertex (together with the associated edges leaving or entering the lost vertex), and persistence will be retained for the remaining graph. For the $C^{2}$ graph with $N$ vertices, there are $2 N$ edges, and one can tolerate the loss of any three edges, or the loss of any vertex and its incident edges, and still retain persistence.

\section{Henneberg sequence theory and persistence graphs}

Given the existence of Henneberg sequence theory for undirected graphs in $\Re^{2}$, it is logical to ask whether the theory can be extended to directed graphs. The topic is treated in [15] and [16]. The broad conclusion is that it can be applied, so long as the primitive operations are modified to allow directed edges in the graphs and also a further primitive operation is introduced. There is more than

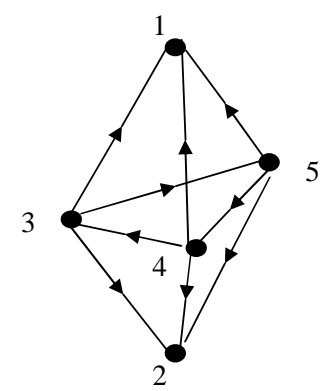

Fig. 6. Illustration of a persistent but not structurally persistent formation

one possible operation, but the simplest possible operation is edge-reversal, i.e., reversing the direction of one edge arriving at a vertex with a degree of freedom (DOF) ${ }^{1}$.

\section{Extension of the persistence concept to $\Re^{3}$}

In $\Re^{3}$, many of the persistence ideas described above will carry through. In particular, one can certainly define constraint consistence and persistence. However, there is a subtle twist. In effect, one needs the equivalent of constraint consistence for all subsets of vertices, as opposed to just each individual vertex considered one at a time. For three and indeed higher dimensions, a concept termed structural persistence is required [36], [37], and in three dimensions, it is very easy to check structural persistence given persistence. Before presenting more details on this concept, we present an example. Figure 6 depicts a three-dimensional formation with an underlying directed graph, and the associated graph is persistent, this being a consequence of every agent having no more than three out-going edges. However, it is evident that agents 1 and 2 are unconstrained, having no out-going edges, and so in principle can move apart, thus destroying the shape of the formation. Hence despite the persistence property, this formation does not have a sensing and control architecture allowing retention of its shape. The reason is that it is not structurally persistent.

Here are now some salient points on persistence and structural persistence for graphs and formations in $\Re^{3}$.

- Any three dimensional graph which has no more than three outgoing edges from any vertex is constraint consistent.

- A graph can be checked for persistence, (i.e. rigidity plus constraint consistence) by testing a certain collection of subgraphs (in which the edge directions are neglected) for rigidity.

Next, if a directed graph is persistent,

- The graph can be checked for structural persistence, which is now the necessary and sufficient condition to be able to provide enough interagent distance controls to ensure the formation behaves as a cohesive whole; for example, structural persistence of a three-dimensional formation can be verified by checking its persistence

\footnotetext{
${ }^{1}$ In $\Re^{2}$, a vertex has two, one or zero degree(s) of freedom if it has no, one, or at least two outgoing edges; each outgoing edge uses up one DOF. A minor variation applies in three dimensions.
} 
and verifying there is at most one vertex of the graph with no outwardly directed edges.

- A three-dimensional persistent graph is always structurally persistent if it is cycle-free.

- It is provable that all persistent graphs in $R^{2}$ and $R^{1}$ are also structural persistent.

- A generalized check for structural persistence can be executed based on the following theorem: a persistent graph in $\Re^{d}(d \geq 1)$ is structurally persistent if and only if every one of its closed subgraphs ${ }^{2}$ with less than $d$ vertices is persistent.

\section{OPERATIONS WITH FORMATIONS}

In [6], several operations involving formations were introduced. In particular, the concepts of splitting, merging and closing ranks were defined, for formations which were modelled using undirected graphs. We give more details:

- Splitting. Consider a single rigid formation. A splitting literately means that its agents are divided into two subsets, and that distance constraints between agents in the different subsets are suppressed. Splitting may occur because of a change of objective, or to avoid an obstacle etc. See Figure 7 for an illustration of the problem. In graph theory terms, after the split, there are two separate (sub-)graphs, neither of which may be rigid. How can one introduce additional distance constraints in the separate subformations to ensure rigidity of them both? Additionally, one can consider variations assuming the starting formation is minimally rigid, one can consider two and three dimensional versions, and one can consider directed graph versions. One can also consider questions of algorithm complexity, and the possibility of posing computational constraints on individual agents if there is a wish to perform calculations on a decentralised basis.

- Merging. Consider two rigid formations. How can additional distance constraints be determined, with one agent in each formation, such that the union of the agents of the two formations, and the union of the distance constraints in the original formations and the new distance constraints, will describe a single rigid formation? Figure 8 illustrates the problem. Of course, the problem can be expressed using graph theoretic terms as well. Again, the problem can be cast in graph theory language, and the same variations apply as for splitting.

- Closing ranks. Consider a single rigid formation. Suppose that one agent is removed, and, consequentially, any distance constraints that applied between this agent and the remaining agents of the formation, see Figure 9. Where should new distance constraints be inserted, in order that the formation can be re-rendered rigid? The same extending remarks apply here also. In addition, the closing ranks problem can be generalised to

${ }^{2} G^{\prime}=\left(V^{\prime}, E^{\prime}\right)$ is a closed subgraph of $G$ if there is no directed path in $G$ starting from $V^{\prime}$ and containing either a vertex or an edge that does not belong to $G^{\prime}$.

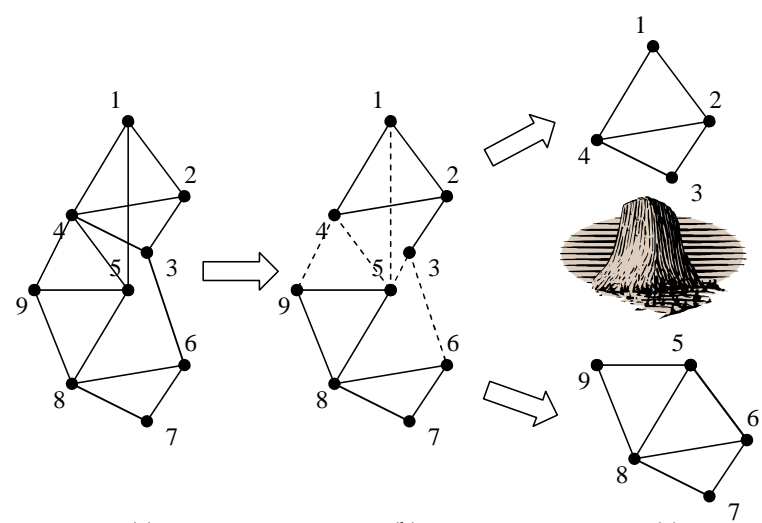

(a)

(b)

(c)

Fig. 7. Illustration of a formation splitting process to avoid obstacles

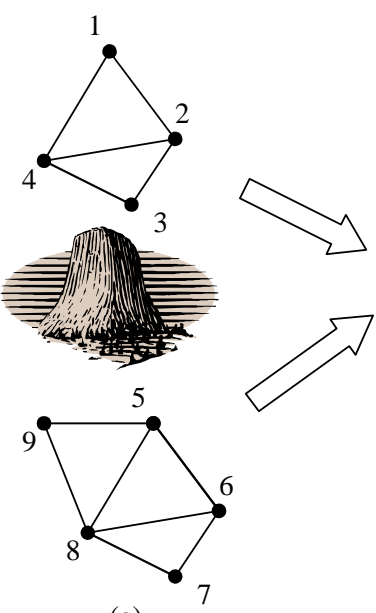

(a)

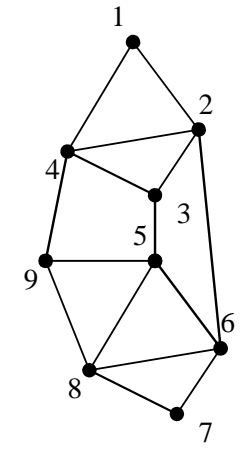

(b)
Fig. 8. Illustration of a merging process

contemplate formations in which more than one agent is simultaneously removed, with the associated distance constraints.

The initial way to solve these problems revolved round finding a significant modification of the Henneberg sequence concept. We describe this in some more detail in the following paragraphs. In the next section, a more recent and different approach is described. In [5], a so-called minimal cover problem was introduced and solved: in the minimal cover problem, a graph is presented which is not minimally rigid. One is required to determine a minimal set of edges (minimality being in the sense of the actual number) which when added to the graph will render it generically rigid. The solution of the minimal cover problem can be applied to solve each of the problems of formatting merging, splitting and closing ranks. Additionally, it has been observed that:

- The splitting problem is actually a particular case of the closing ranks problem. One subformation can regard the agents of the other subformation as the lost agents.

- The closing ranks problem (in graph theoretic language) can always be solved by introducing new edges between former neighbours of the lost vertices of the graph, i.e. 


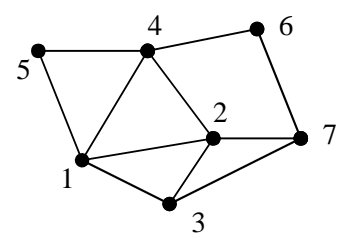

(a)

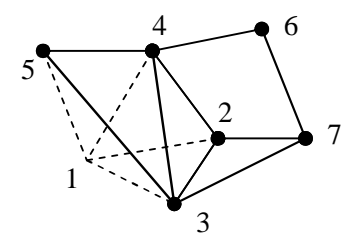

(b)
Fig. 9. Illustration of a closing rank process where agent 1 and all associated links are lost, and new links $(5,3)$ and $(4,3)$ are inserted to reestablish the rigidity. Agents 6 and 7 are not affected during the process.

by performing a local repair as illustrated in Figure 9. In connection with the splitting problem, this means that any new edges can be restricted to connecting pairs of those vertices in one subformation graph that were previously neighbours of vertices which ended up in the other subformation graph, as illustrated in Figure 7.

The above formation operations can also be contemplated for directed graphs. However, little work has so far been done.

\section{META-VERTICES, BODIES AND META-FORMATIONS}

In merging two formations, it is obvious that much internal structure is largely irrelevant. As it turns out for example, if two rigid formations are to be merged in two dimensions, this can always be done by introducing three distance constraints with one agent in each of the two formations, and ensuring that in each of the two formations, at least two agents are involved in the distance constraints [34]. There is obviously some kind of a general rule operative here and it is of interest to establish what the general rules are concerning the connection of formations to form larger formations, particularly ensuring preservation of rigidity; with the view however that the internal connections of the individual formations are unimportant, we shall term the larger formation a metaformation [1], [35]. In this connection, we shall first note two streams of work.

\section{A. Rigidity and two-dimensional formations of formations}

The papers [27], [30] investigate what are sometimes termed body-bar systems. A body is like a generalization of a point agent. Any rigid formation of agents can be replaced by a body, a rigid object that in two dimensions has three degrees of freedom, two displacements and one rotation. (In contrast, a point agent in two dimensions has two degrees of freedom, both translational). Each body can be deemed to have a set of connection points on its surface, with the property that distances can be constrained between two connection points in different bodies. One can imagine a formation comprising a set of bodies, which might also be termed meta-vertices or meta-agents, with certain distance constraints between them (usually more than one connection point on the surface of a body is used; for if only one connection point were used, the body or the meta-vertex could rotate about it). The term meta-vertex is however probably best restricted to applying to the graph equivalent of a body. One can also pose the question: when will such a formation be rigid? Of course, it is desired to answer this question taking no account of the internal structure of the bodies.

The question was answered for meta-formations of bodies in [27], [30], using both a generalization of the rigidity matrix, and a generalization of Laman's Theorem for the two-dimensional case. Recall that Laman's Theorem, see Appendix B, provides necessary and sufficient conditions for generic rigidity of a graph corresponding to a formation of point agents, and the conditions are of a "counting" form; a simple adjustment of certain numbers appearing in the statement of Laman's Theorem converts it to a theorem concerning generic rigidity of a graph corresponding to a twodimensional bar-body framework. As for checking rigidity of a normal graph in $\Re^{3}$, the available counting condition for three-dimensional body-bar framework are necessary for it to be rigid but not sufficient. The rigidity matrix ideas work in three dimensions (where the bodies are three dimensional and thus have six degrees of freedom, three translational ones and three rotational ones) [30].

Interconnection of two formations is a matter of interconnection of two bodies, and the Laman's Theorem extension easily provides the result that three distance constraints between connection points on each of the two bodies, with at least two connection points involved for each body, serves to give rigidity of the overall formation. This idea could be extended in that of merging more than two formations (metavertices) and agents (vertices) [35]. This type of result of course exists for directed graphs, some results are available in [17].

\section{B. More on formation merging}

A recent paper [34] by some of the authors considers the problem described above of connecting two formations in two dimensions as well as other problems:

- Connecting (via insertion of additional edges) two formations in three dimensions to secure minimal rigidity.

- Connecting two formations in two or three dimensions to secure global rigidity.

- Connecting two formations when they are not disjoint, i.e., they are permitted to have a limited number of common vertices and/or a limited number of common edges.

By appealing to various results on rigidity and global rigidity, a series of conditions are established to solve these problems. The conditions are generally of the form: make $m$ connections, involve at least $n$ vertices of one formation, and at least $p$ vertices of the second formation. We give two examples, to make more concrete the form of the results.

- Consider two globally rigid two-dimensional graphs, with one vertex in common. Then by adding two new edges with one vertex in each formation, and such that in at least one of the formations the edges are incident on two different vertices, a globally rigid graph results. (Note that the two new edges added cannot be incident on the vertex common to the two initially given graphs) 
- Consider two minimally rigid three dimensional graphs, with no vertices in common. Then after addition of six new edges, incident in each graph on at least three vertices, and with no more than two edges incident to a single vertex, there results a minimally rigid graph. A related result is that if the two three-dimensional graphs are rigid but not necessarily minimally rigid, addition of six new edges using the same incidence rules will result in a rigid graph, but fewer than six edges cannot. We might regard such an interconnection as minimally rigid from the meta-formation point of view, since the issue of whether or not the individual meta-vertices (themselves formations) are minimally rigid (they must of course be rigid) is considered irrelevant.

Directed versions of these results have in part been obtained [17]. Of course, the conditions for securing persistence always include those applicable to securing rigidity, as discussed before. Here are some examples of conclusions which can be established:

- In order to merge two minimally persistent graphs (in $\Re^{3}$ ) into a larger minimally persistent graph, one needs to add six directed interconnection edges that leave vertices with some degrees of freedom in the initial (premerging) graphs (one DOF for each out-going edge) but that can arrive at any vertices of the other initial (pre-merging) graphs. (In $\Re^{3}$, the degrees of freedom of a vertex are three, two, one or zero, according as the vertex has respectively no, one, two, three or more outgoing edges.) Not every selection of interconnection edges leads to a persistent merged graph, but it is always possible to find a set of interconnection edges that makes the merged graph structurally persistent, even when the initial (persistent) graphs are not structurally persistent.

- When the merged graph needs to be persistent and not necessarily minimally persistent, one still needs to add six directed edges leaving vertices with some (positive) degrees of freedom. The number of new edges leaving a vertex with positive degree of freedom must be no greater than its degree of freedom. Other edges (possibly leaving vertices without DOF) can under some conditions also be added, but they can always be avoided. As a consequence, at least six degrees of freedom must be available in the two initial graphs; otherwise the two graphs cannot be merged.

- If one of the two initial graphs has no degree of freedom and if simultaneously the other one is not structurally persistent, then they cannot be merged into a persistent graph. However, in every other case, if six degrees of freedom are available, it is always possible to choose six directed interconnection edges to make the merged graph persistent and even structurally persistent.

We have further defined two types of persistent merging, as depicted in Figure 10:

- (Meta-)Leader-Follower merging, in which all newly added directed edges leave from a persistent formation

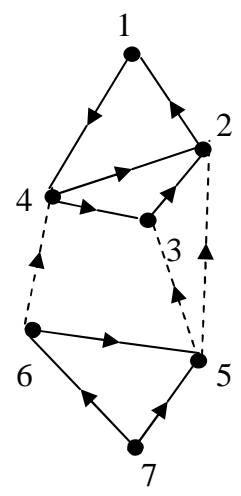

(a)

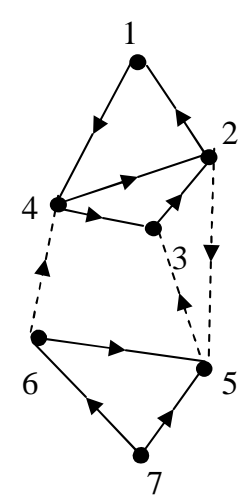

(b)
Fig. 10. Illustration of (a) Leader-Follower merging, and (b) Collaborative merging. Formation $G_{1}$ consisting of agents 5, 6 and 7, and formation $G_{2}$ consisting of agents $1,2,3$, and 4 .

$G_{1}$ (the "meta-follower") and arrives at $G_{2}$ (the "metaleader"). These directed meta-edges therefore remove all DOFs of $G_{1}$ and the merged persistent formation retains the same DOF allocation pattern of $G_{2}$.

- Collaborative merging, in which each formation will have some newly added directed edges leaving one or more of their vertices with positive DOF, and arriving at the other formation. Evidently, both formations will lose some DOF, and as a result, the DOF allocation pattern of the post-merged persistent formation may be different from that of either $G_{1}$ or $G_{2}$.

Some results related to the above characterizations of merging persistent formations have been noted and some are given below:

- Collaborative merging in the three-dimensional case may produce a formation that is not structurally persistent, even if the two pre-merging formations are structurally persistent. This happens when both initial formations contain a leader, and when none of the edges added during the merging process leaves either of those two leaders.

- In the case of a leader-follower merging, where the pre-merging formations contain more than just a single agent, the formation obtained is always structurally persistent.

\section{Towards a more systematic theory}

Given that in $\Re^{2}$ one can find a version of Laman's Theorem describing the rigidity of a meta-formation, obtained by connecting together meta-vertices or meta-agents, one can also ask: is there a concept of a Henneberg sequence for meta-formations? Such a sequence could start with a single meta-vertex, or rigid formation, and involve the successive addition of meta-agents to the meta-formation. Each addition would result in a meta-formation that had the minimal number of edges between meta-vertices so as to guarantee rigidity of the overall meta-formation. Indeed, that is the case. Analogs to vertex addition and edge splitting, termed 


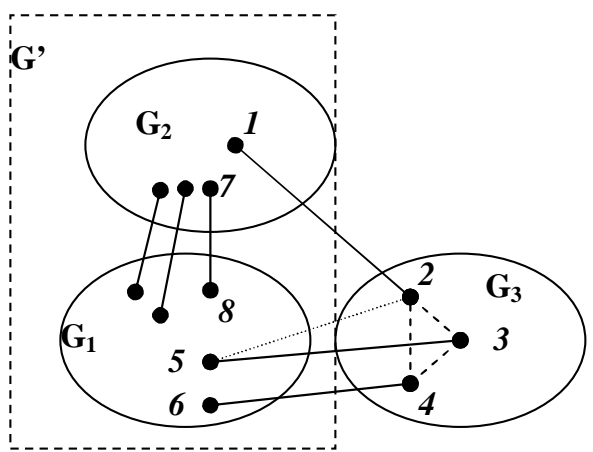

(a)

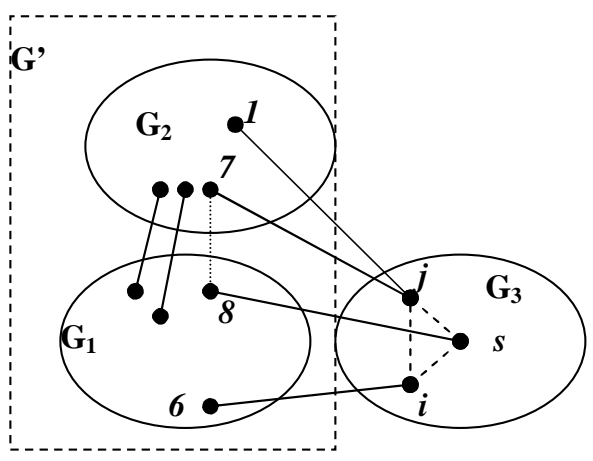

(b)

Fig. 11. Illustration of a scenario when one can perform meta-Henneberg operations: Meta-vertices $G_{1}$ and $G_{2}$ are merged into $G^{\prime}$ with three metaedges, meta-vertex $G_{3}$ is merged using (a) meta-vertex addition and (b) meta-edge splitting.

meta-vertex addition and meta-edge splitting respectively, can be constructed, see for example Fig. 11. The process can also be described by building on the results described in the previous section, and was set out in detail in our recent work [35].

\section{CONCLUSiOnS}

In this paper, we have set out the rudiments of a theory for analysing and creating architectures appropriate to the control of formations of autonomous vehicles. The theory rests on ideas of rigid graph theory, some but not all of which are old. The theory however has a number of gaps in it, and their elimination would help in applications. Some of the gaps in the relevant graph theory are as follows:

- There is as yet no analog for three-dimensional graphs of Laman's theorem, which provides for twodimensional graphs a combinatorial criterion for rigidity

- For three dimensional graphs there is no analog of the two-dimensional Henneberg construction (although there are conjectures, [31])

- Global rigidity can be easily characterized for twodimensional graphs; this is not the case for threedimensional graphs.

Actually, it may be the case that one will need to study graphs in dimensions higher than 3 , because the physical agents they are modelling may have orientation as well as position. Generally speaking, results for dimensions higher than three are even less well documented.

Turning more to the applications, let us note the following important questions:

- What is a satisfactory measure of redundancy in a formation, i.e. a measure of its ability to sustain loss of agents or communication/control links?

- For a given number of agents, and with a constraint on the number of edges in the associated graph, what formation shapes in $\Re^{2}$ and $\Re^{3}$ are the most robust to edge and/or agent loss? Are there any trade-offs?

- What is a complete description of the set of distributed control laws that can be used to maintain the shape of a formation, both in the undirected and the directed graph case (a preliminary question is: what is a control law which will maintain the shape of a formation-but this question is now well answered for formations modelled by undirected graphs-need references-and the work of Lee and Spong [24] deals with directed graphs). Of course, this question is a logical follow-on to the ideas of this paper that must be addressed in order that the ideas can be operationalised.

- What formations are going to be difficult to control, in the sense of requiring very accurate sensors and/or very large control signals? Might they be those for which the rigidity matrix has its smallest nonzero singular value close to zero?

- What variations to the results apply when geometric information other than distances is sensed, such as, for example, angles, differences of distances (due to Time Difference of Arrival information)?

- What variations of the results apply when orientation of agents is relevant?

\section{REFERENCES}

[1] B.D.O. Anderson, C. Yu, B. Fidan, and J.M. Hendrickx. Use of metaformations for cooperative control. To appear in the 17th International Symposium on Mathematical Theory of Networks and Systems, July 2006.

[2] J. Baillieul and A. Suri. Information patterns and hedging brockett's theorem in controlling vehicle formations. In Proc. of the 42nd IEEE Conf. on Decision and Control, volume 1, pages 556-563, December 2003.

[3] M. Cao, A.S. Morse, and B.D.O. Anderson. Sensor network localization with imprecise distances. System and Control Letters, to appear.

[4] A. Das, R. Fierro, V. Kumar, and J.P. Ostrowski. A vision-based formation control framework. IEEE Trans. on Robotics and Automation, 18(5):813-825, October 2002.

[5] T. Eren, B.D.O. Anderson, A.S. Morse, W. Whiteley, and P.N. Belhumeur. Information structures to control formation splitting and merging. In Proc. of the American Control Conference, 2004.

[6] T. Eren, B.D.O. Anderson, A.S. Morse, W. Whiteley, and P.N. Belhumeur. Operations on rigid formations of autonoumous agents. Communications in Information and Systems, pages 223-258, September 2004.

[7] T. Eren, B.D.O. Anderson, W. Whiteley, A.S. Morse, and P.N. Belhumeur. Merging globally rigid formations of mobile autonomous agents. In Proc. of the AAMAS, 2004.

[8] T Eren, D. Goldenberg, W Whiteley, Y.R. Yang, A.S. Morse, B.D.O. Anderson, and P.N. Belhumeur. Rigidity, computation, and randomization in network localization. In Proc. of the International Annual Joint Conference of the IEEE Computer and Communications Societies, pages 2673-2684, Hong Kong, March 2004. 
[9] T. Eren, W. Whiteley, A. S. Morse, P. N. Belhumeur, and B. D.O. Anderson. Sensor and network topologies of formations with direction, bearing and angle information between agents. Proceedings of the 42nd IEEE Conference on Decision and Control, pages 3064-3069, December 2003.

[10] B. Fidan, C. Yu, and B.D.O. Anderson. Acquiring and maintaining persistence of autonomous multi-vehicle formations. IEE Proc. Control Theory and Applications, 2006 to appear.

[11] V. Gazi and K.M. Passino. Stability analysis of swarms. IEEE Trans. on Automatic Control, 48(4):692-697, April 2003.

[12] D.K. Goldenberg, A. Krishnamurthy, W.C. Maness, Y.R. Yang, A. Young, A.S. Morse, A. Savvides, and B.D.O. Anderson. Network localization in partially localizable networks. In Proceedings of Infocom 05', Miami, March 2005.

[13] J.M. Hendrickx, B.D.O. Anderson, J.-C. Delvenne, and V.D. Blondel. Directed graphs for the analysis of rigidity and persistence in autonomous agent systems. International Journal of Robust Nonlinear Control, 2006 to appear.

[14] J.M. Hendrickx, B. Fidan, C. Yu, B.D.O. Anderson, and V.D. Blondel. Rigidity and persistence of three and higher dimensional directed formations. In Proc. of 1st International Workshop on Multi-Agent Robotic Systems, Barcelona, Spain, September 2005.

[15] J.M. Hendrickx, B. Fidan, C. Yu, B.D.O. Anderson, and V.D. Blondel. Elementary operations for the reorganization of peristent formations. To appear in the 17th International Symposium on Mathematical Theory of Networks and Systems, July 2006.

[16] J.M. Hendrickx, B. Fidan, C. Yu, B.D.O. Anderson, and V.D. Blondel. Primitive operations for the construction and reorganization of minimally persistent formations. Submitted for publication, 2006.

[17] J.M. Hendrickx, C. Yu, B. Fidan, and B.D.O. Anderson. Rigidity and persistence of meta-formations. Submitted for publication, 2006.

[18] L. Henneberg. Die graphische Statik der starren Systeme. Leipzig, 1911.

[19] S. Hubbard, B. Babak, S.T. Sigurdsson, and K.G. Magnusson. A model of the formation of fish schools and migrations of fish. Ecological Modelling, 174:359-374, 2004.

[20] B. Jackson and T. Jordan. Connected rigidity matroids and unique realizations of graphs. Journal of Combinatorial Theory, B(94):1-29, 2004.

[21] A. Jadbabaie, J. Lin, and A. S. Morse. Coordination of groups of mobile autonomous agents using nearest neighbor rules. IEEE Trans. on Automatic Control, 48(6):988-1001, June 2003.

[22] S. Janson, M. Middendorf, and M. Beekman. Honey bee swarms: How do scouts guide a swarm of uninformed bees? Animal Behaviour, 70(1):349-358, 2005.

[23] G. Laman. On graphs and rigidity of plane skeletal structures. $J$. Engrg. Math., 4:331-340, 1970.

[24] D.J. Lee and M.W. Spong. Stable flocking of inertial agents on balanced communication graphs. To appear in the American Control Conference, 2006.

[25] Z. Lin, B.A. Francis, and M. Maggiore. Necessary and sufficient graphical conditions for formation control of unicycles. IEEE Trans. on Automatic Control, 50(1):121-127, January 2005.

[26] D. Moore, J. Leonard, D. Rus, and S. Teller. Robust distributed network localization with noisy range measurements. pages 50-61, Baltimore, MD, November 2004.

[27] C. Moukarzel. An efficient algorithm for testing the generic rigidity of graphs in the plane. J. Phys. A: Math. Gen., (29):8079-8098, 1996.

[28] R. Olfati-Saber and R. M. Murray. Distributed cooperative control of multiple vehicle formations using structural potential functions. Proc. of the 15th IFAC World Congress, 2002.

[29] H.G. Tanner, G.J. Pappas, and V. Kumar. Leader-to-formation stability. IEEE Transactions on Robotics and Automation, 20(3):443-455, June 2004.

[30] T. Tay. Rigidity of multigraphs, i: linking rigid bodies in n-space. $J$. Combinat. Theory B, (36):95-112, 1985.

[31] T. Tay and W. Whiteley. Generating isostatic frameworks. Structural Topology, (11):21-69, 1985.

[32] W. Whiteley. Matroid Theory, volume 197 of Contemporary Mathematics, chapter Some Matroids from Discrete Applied Geometry, pages 171-311. American Mathemtical Society, 1996.

[33] C. Yu, B. Fidan, and B.D.O. Anderson. Persistence acquisition and maintenance for autonomous formations. In Proc. of the 2 nd International Conference on Intelligent Sensors, Sensor Networks and Information Processing, Melbourne, Australia, 2005.
[34] C. Yu, B. Fidan, and B.D.O. Anderson. Principles to control autonomous formation merging. In American Control Conference, Minneapolis, MN, June 2006 to appear.

[35] C. Yu, B. Fidan, J.M. Hendrickx, and B.D.O. Anderson. Merging multiple formations: A meta-formation prospective. Submitted for publication, 2006.

[36] C. Yu, J.M. Hendrickx, B. Fidan, and B.D.O. Anderson. Structural persistence of three-dimensional autonomous formations. In Proc. of 1 st International Workshop on Multi-Agent Robotic Systems, Barcelona, Spain, September 2005.

[37] C. Yu, J.M. Hendrickx, B. Fidan, B.D.O. Anderson, and V.D. Blondel. Three and higher dimensional autonomous formations: Rigidity, persistence and structural persistence. Automatica, 2006 to appear.

\section{APPENDICES}

\section{A. Rigidity matrices [31]}

Consider a graph $G=(V, E)$ in $\Re^{2}$ of $|V|$ vertices and $|E|$ edges. Let the coordinates of vertex $j$ be $\left(x_{j}, y_{j}\right)$. The rigidity matrix is defined with an arbitrary ordering of the vertices and edges, and has $2|V|$ columns and $|E|$ rows. Each edge gives rise to a row, and if the edge links vertices $j$ and $k$, the nonzero entries of the row of the matrix are in columns $2 j, 2 j+1,2 k$ and $2 k+1$, and are respectively $x_{j}-x_{k}$, $y_{j}-y_{k}, x_{k}-x_{j}, y_{k}-y_{j}$. For example, for the graphs of Figure 1(a) and 1(c), the rigidity matrices are

$$
\left[\begin{array}{cccccccc}
x_{1}-x_{2} & y_{1}-y_{2} & x_{2}-x_{1} & y_{2}-y_{1} & 0 & 0 & 0 & 0 \\
0 & 0 & x_{2}-x_{3} & y_{2}-y_{3} & x_{3}-x_{2} & y_{3}-y_{2} & 0 & 0 \\
0 & 0 & 0 & 0 & x_{3}-x_{4} & y_{3}-y_{4} & x_{4}-x_{3} & y_{4}-y_{3} \\
x_{1}-x_{4} & y_{1}-y_{4} & 0 & 0 & 0 & 0 & x_{4}-x_{1} & y_{4}-y_{1}
\end{array}\right]
$$

and

$$
\left[\begin{array}{cccccccc}
x_{1}-x_{2} & y_{1}-y_{2} & x_{2}-x_{1} & y_{2}-y_{1} & 0 & 0 & 0 & 0 \\
0 & 0 & x_{2}-x_{3} & y_{2}-y_{3} & x_{3}-x_{2} & y_{3}-y_{2} & 0 & 0 \\
0 & 0 & 0 & 0 & x_{3}-x_{4} & y_{3}-y_{4} & x_{4}-x_{3} & y_{4}-y_{3} \\
x_{1}-x_{4} & y_{1}-y_{4} & 0 & 0 & 0 & 0 & x_{4}-x_{1} & y_{4}-y_{1} \\
x_{1}-x_{3} & y_{1}-y_{3} & 0 & 0 & x_{3}-x_{1} & y_{3}-y_{1} & 0 & 0 \\
0 & 0 & x_{2}-x_{4} & y_{2}-y_{4} & 0 & 0 & x_{4}-x_{2} & y_{4}-y_{2}
\end{array}\right]
$$

There can be special positions for the vertices of a graph where rigidity is lost, typically when vertices are collinear in $\Re^{2}$ or coplanar in $\Re^{3}$. Call such situations non-generic. The key result is:

Theorem: A graph $G=(V, E)$ in $\Re^{2}$ of $|V|$ vertices and $|E|$ edges is rigid if and only if for generic vertex positions, the rigidity matrix has rank $2|V|-3$.

It is easy to verify this result for the two examples. The rank for the rigidity matrix of Figure 1(a) is 4 and for Figure 1 (c) it is 5 .

In case the rigidity matrix has rank $2|V|-3$, the dimension of the kernel is 3 . It is possible to argue that independent vectors in the kernel correspond to agent velocities when the formation is translating and/or rotating. In two dimensions, there are two independent translations and one rotation which are possible. When the kernel dimension is greater than 3 , independent motions in addition to translation and rotation are possible, corresponding to some kind of flexing.

The theorem extends easily to graphs in $\Re^{3}$. There are now three columns of the matrix associated with each vertex and 
rigidity for generic vertex positions corresponds to having a rank of $3|V|-6$.

The rigidity matrix of a physical structure conveys information about how loads on a structure translate into forces in its members. Rigidity matrices with three zero singular values and with a very small nonzero singular value appear to correspond to physical structures which could be considered to not be very rigid, i.e. small loads could cause large deflections.

\section{B. Laman's theorem [23]}

Laman's theorem requires the idea of an induced subgraph of a graph $G=(V, E)$. Let $V^{\prime}$ be a subset of $V$. Then the subgraph of $G$ induced by $V^{\prime}$ is the graph $G^{\prime}=\left(V^{\prime} E^{\prime}\right)$ where $E^{\prime}$ includes all those edges of $E$ which are incident on a vertex pair in $V^{\prime}$.

Laman's Theorem: A graph $G=(V, E)$ in $\Re^{2}$ of $|V|$ vertices and $|E|$ edges is rigid if and only if there exists a subgraph $G^{\prime}=\left(V, E^{\prime}\right)$ with $2|V|-3$ edges such that for any subset $V$ " of $V$, the induced subgraph $G "=(V ", E ")$ of $G^{\prime}$ obeys $\left|E^{\prime \prime}\right| \leq 2\left|V^{\prime \prime}\right|-3$.

Partial extension of Laman's theorem to $\Re^{3}$ : A graph $G=$ $(V, E)$ in $\Re^{3}$ of $|V|$ vertices and $|E|$ edges is rigid only if (a) there exists a subgraph $G^{\prime}=\left(V, E^{\prime}\right)$ with $3|V|-6$ edges such that for any subset $V^{\prime \prime}$ of $V$, the induced subgraph $G "=(V ", E ")$ of $G^{\prime}$ obeys $|E "| \leq 3\left|V^{\prime \prime}\right|-6$, and (b) if $G$ " obeys $\left|E^{\prime \prime}\right|=3\left|V^{\prime \prime}\right|-6$, then it is 3-connected, i.e. between any two vertices of $G$ ", there are three paths which pairwise have no vertices in common.

\section{Henneberg Constructions [18]}

Let $G=(V, E)$ be a graph in $\Re^{2}$. The operation of vertex addition is the following. A new graph $G^{\prime}=\left(V^{\prime}, E^{\prime}\right)$ is formed in which a vertex $v$ is adjoined as well as two edges from $G$ to $v$, so that $V^{\prime}=V \cup v$ and $E^{\prime}=E \cup(v, j),(v, k)$ for $j, k \in V$.
The operation of edge splitting is the following. A new graph $G^{\prime}=\left(V^{\prime}, E^{\prime}\right)$ is formed in which a vertex $v$ is adjoined as well as three edges from $G$ to $v$, while an edge of $G$ is removed. More precisely, $V^{\prime}=V \cup v$ and $E^{\prime}=E \cup(v, j),(v, k),(v, m) \backslash e$ for $j, k, m \in V$ with at least two of $j, k, m$ adjacent in $G$, and $e$ one of $(j, k),(j, m)$, or $(k, m)$.

\section{Global Rigidity Characterization}

For two-dimensional formations and their associated graphs, there is a nice characterization of global rigidity. (No extension is known for three-dimensional formations.). Call a graph redundantly rigid if it remains rigid after the removal of any single edge.

Theorem: A graph $G=(V, E)$ in $\Re^{2}$ of $|V|$ vertices and $|E|$ edges is globally rigid if and only if it is redundantly rigid and 3-connected.

\section{E. Persistence Testing}

As noted in the main body of the text, there is a test for persistence of a graph. It runs as follows in $\Re^{2}$. Let $G$ be a directed graph, and let $\left\{G_{i}, i=1,2, \ldots\right\}$ be the set of undirected graphs obtainable from $G$ by deleting edges outgoing from any vertex with out-degree exceeding 2 , until there are just two outgoing edges. (If there are for example, three vertices in $G$ with out-degree 3 and two with out-degree 4 , the total number of graphs $G_{i}$ will be $(3)^{3}(6)^{2}$, being three possible ways of selecting two outgoing edges from three, for each of three vertices, and six possible ways of selecting two out going edges from four, for each of two vertices.) Then $G$ is persistent if and only if all undirected versions of the $G_{i}$ are all persistent.

In $\Re^{3}$, the same idea applies, except that outgoing edges in excess of 3 rather than 2 are deleted. 